University of San Diego

Digital USD

Spring 5-17-2016

\title{
Tropical Trees as Islands: Diversity Accumulation of Armored Scale Insects (Hemiptera: Diaspididae) on Trees as a Function of Forest Age
}

Hannah Shapiro

University of San Diego

Follow this and additional works at: https://digital.sandiego.edu/honors_theses

Part of the Biodiversity Commons, Bioinformatics Commons, Entomology Commons, Evolution Commons, Genetics Commons, Genomics Commons, Molecular Genetics Commons, and the Population Biology Commons

\section{Digital USD Citation}

Shapiro, Hannah, "Tropical Trees as Islands: Diversity Accumulation of Armored Scale Insects (Hemiptera: Diaspididae) on Trees as a Function of Forest Age" (2016). Undergraduate Honors Theses. 63.

https://digital.sandiego.edu/honors_theses/63

This Undergraduate Honors Thesis is brought to you for free and open access by the Theses and Dissertations at Digital USD. It has been accepted for inclusion in Undergraduate Honors Theses by an authorized administrator of Digital USD. For more information, please contact digital@sandiego.edu. 
Tropical Trees as Islands: Diversity Accumulation of Armored Scale Insects

(Hemiptera: Diaspididae) on Trees as a Function of Forest Age

\author{
A Thesis \\ Presented to \\ The Faculty and the Honors Program \\ Of the University of San Diego
}

By

Hannah Rochelle Shapiro

Department of Biology

2016 


\section{ABSTRACT}

Armored scale insects (Hemiptera: Diaspididae) are some of the most invasive insects in the world. These cryptic plant parasites are most often encountered in managed agricultural ecosystems, but very little is known about their distribution, abundance, and diversity in tropical rainforest canopies, where they are likely to have their highest diversity. Because these ubiquitous insects are extreme generalists with undirected dispersal, their diversity (alpha and beta) accumulation can conceivably be modeled according to tenets derived from island biogeography theory. For example, one expectation is that older established trees should boast a higher species diversity and abundance than younger ones. Other aspects of island biogeography theory have been unexplored, especially in regards to expected phylogenetic patterns of community structure. In this study we combine an intense ecological survey with a community phylogenetics approach to examine these unexplored aspects. Specimens were collected in an intensive survey from both (1) a tropical rainforest canopy using a canopy access crane in the Daintree Rainforest in Australia; and (2) an adjacent five year-old reforestation plot that had a similar diversity and constituency as the mature plot. We use a combination of systematic environmental sampling and molecular and morphological species delimitation techniques to compare and contrast the abundance, diversity, and community phylogenetics of these two habitats in order to address this question. 


\section{BACKGROUND \& INTRODUCTION}

\section{Armored Scale Insect Characterization}

Armored scale insects (Hemiptera:Diaspididae) are some of the most invasive and bizarre plant parasites in the world (Anderson et. al. 2010). Their cryptic nature and extremely small size (on the order of 200 microns) allows them to settle and feed on virtually any exposed portion of the plant. To date, at least 8,194 species have been described within 50 families (Morales et. al. 2016), though most of what we know about armored scale insects comes from agricultural or horticultural systems, where we most frequently encounter them. Armored scale insects are ubiquitous, having been characterized on every continent, except for Antarctica, with the capability of reaching extreme population sizes on individual hosts, a characteristic notorious to invasive pests. These miniscule organisms frequently evade quarantine and become invasive through global trade of plant material (Anderson et. al. 2010). Although we are becoming more familiar with these pests in an agrarian setting, we know very little about them in natural settings such as tropical rainforests, where they are believed to have actually originated.

\section{Armored Scale Insect Life History and Biology}

Although they are known as "crawlers" in their larval stage, first-instars are winddispersed and are capable of minimal mobility once they land on plant material. Females will settle and begin feeding on the contents of parenchyma cells using their stylet-like mouthpart. As second instars, they lose their eyes, legs, and antennae, becoming completely sessile for the duration of their lives (Morse \& Normark 2006). Such extremely reduced morphology as exhibited in adult females is a feature commonly seen in polyphagous insect pest species. They secrete a hard, shell-like covering over their dorsal aspect, giving them the name "armored scale 
insects." This cuticle incorporates plant matter, the exuviae of the previous instar, and a waxy, hydrophobic substance, which serves as protection and sealant to the plant material. Only their reproductive pygidium is exposed so that males can copulate. Because they are stationary, adult females are the most frequently encountered life stage, though their extremely reduced morphology makes identification exceedingly difficult, if not impossible, using alpha-taxonomy alone (Morse \& Normark 2006).

These insects exhibit an unusually high degree of sexual dimorphism, as male armored scale insects have three non-feeding instar stages and are winged as adults (Gullan and Kosztarab 1997). Lacking mouthparts as adults, males do not feed in their winged stage, and only live for about three days. They must therefore locate a female and copulate with her within this relatively short time-frame, in order to successfully reproduce.

\section{Unusual Genetic Systems}

Along with a high degree of sexual dimorphism and a bizarre life history, armored scale insects exhibit some of the most diverse genetic systems seen in insects. Male armored scale insects frequently undergo early or late paternal genome elimination (PGE). Both sexes undergo genetic chimerism, in which their bacteriocytes form from polar bodies. Within these bacteriocytes, endosymbiotic bacteria are housed (Normark 2004). Normark has identified a wide breadth of unusual genetic systems, many of which are unstable, in armored scale insects, including variability in both ploidy and the constituents of bacteriomes (Normark 2004).

\section{Extreme polyphagy}

It is speculated that armored scale insects are among the very few insect groups that exhibit apparent host-generalism. Some of the features that define well known insect pests 
include woody host plants usage, flightless adult females, larval dispersal by wind or via soil, extreme population sizes and abundances, and recognition as a common pest. Often, we find that invasive pest species are capable of maintain parthenogenic populations with a coinciding expansive geographical range (Normark \& Johnson 2011). Armored scales have most, if not all, of these features, and are commonly recognized as pests. But are armored scale insects truly generalists? While most insects have an exceptionally narrow range of coevolution with a single host species, or even a few hosts among genera, it was found that 286 armored scale insect species are able to feed on 20 or more families of hosts (Normark \& Johnson 2011). Alongside Noctuid (Noctuidae) moths, soft-scales (Coccidae), and aphids (Aphidae), Diaspidids account for 59 of the species on the list of invasive insect taxa; this is approximately double that of any other family of insects on this list. This generalism is peculiar in the insect world, and thus it has been speculated that this apparent generalism is merely an illusion, masking what is actually a complex assemblage of cryptic species. However, there is a great deal of support for their generalism, most notably, their ability to thrive in both native and non-native habitats and their ability to use a vast multitude of hosts (Miller et. al. 2005).

Though this study is not focused on the apparent generalism of armored scale insects, it is a unique feature that might help to explain factors underlying their community composition in two habitats. The uniquely sessile nature of the adult female phase is convenient for record keeping; because an adult female spends her entire life feeding on a single host, the collection of a single adult individual represents usage of a single host species throughout her entire life. Therefore, the "tourist" problem does not come into play here, as it does in many other ecological surveys; data supporting evidence of wide host usage can therefore not be dismissed as merely an artifact (Novotny et al. 2002). 


\section{The Niche Explosion Hypothesis}

The Niche Explosion Hypothesis (Normark \& Johnson 2011) was proposed as an explanation for the apparent generalism observed in armored scale insects. With wind-dispersed larvae, nearly all woody plants in a given area are subject to being "rained down upon" and settled on by larvae. It is proposed that because of their lack of directional-dispersal, natural selection confers upon them the ability to utilize a wide variety of hosts (essentially, they must use the host they land on, or else, they will not survive). With exponentially large population sizes on a single host and wind dispersal capabilities, a positive feedback loop is generated. As host species usage grows, more "infected" individuals accumulate per each host species, and new hosts are colonized more frequently. As natural selection acts more forcibly upon large population sizes, this confers a selective advantage for the accumulation of genetic loci that provide armored scales with the ability to maintain themselves on a wide variety of hosts. Because of this, population size, host range, and host usage expand markedly, giving it the name "niche explosion" (Normark \& Johnson 2011).

\section{Economic importance}

While these nearly "microscopic" organisms are not particularly charismatic, aside from their fascinating genetic systems and reproduction, they have extreme economic importance. Most of what we know about their biology stems from their collection from agricultural crops, where they can reach devastatingly large populations. The destruction caused by armored scale insects is estimated to cost two billion dollars per year in the United States due to the damage they cause to orchards, forests, agriculture and horticulture (Miller et. al. 2005). Moreover, these insects are among the most invasive of crop pests. In 2005, $40 \%$ of all known armored scale 
fauna in the United States were found to be introduced from other countries, at a species estimate of 132, in total. It is estimated that around one new species of armored scale insect is introduced into the United States every year, a number that, when compounded, could have devastating effects on crops (Miller et al. 2005).

\section{The Island Biogeography Hypothesis}

After a series of ecological experiments in benthic marine communities, ecologists E. O. Wilson and R. H. MacArthur developed the island biogeography hypothesis (1967), which attempted to identify the factors that influence species diversity in island habitats. Their theory refers to "islands" in both the traditional sense, as well as a "nontraditional" sense, which would include fragmented or isolated terrestrial habitats. Factors influencing species richness and abundance are the degree of isolation to neighboring communities or to the mainland, the length of time for which it is isolated, the habitat area, human influence, and pure luck! The time component is particularly relevant to the study of armored scale insects in the Australian rainforest. This component, further explained by the "time-stability hypothesis" coined by H.L. Sanders (1969), predicts that older habitats will generally have a greater species richness and diversity than younger habitats with similar host constituency. Moreover, because older habitats are generally more stable, competitive species interactions more frequently dominate in these communities, resulting in a high species diversity and narrower niche space. Thus, younger habitats, which are less likely to have reached stability, should have a lower species diversity and broader niche space (Abele \& Walters 2016) (Sanders 1969). Based on this hypothesis, armored scale insect diversity should be higher in an older rainforest canopy, as compared to a 5-year old revegetation plot. This component of island biogeography was used to model a predicted pattern 
of armored scale diversity, given that these insects have never before been collected in the Australian rainforest.

Community Ecology and Phylobetadiversity

The objective of this experiment was to determine whether or not the principles of island biogeography theory and the time-stability hypothesis (Sanders 1969) can be used to describe the alpha and beta diversity of armored scale insects in a tropical rainforest. In recent years, the study of community ecology has become increasingly integrated with the field of phylogenetics; this has created new avenues of study and has provided us with new tools that allow us to address traditional questions of community ecology using an evolutionary approach (Cavender et. al. 2009). Phylobetadiversity is therefore directly relevant to this study and is used to approach complex questions about armored scale insect biodiversity in a tropical rainforest using phylogenetic, as well as community composition data.

In this study, we seek to integrate phylogenetics with concepts of community ecology for a comparative analysis. This approach, known as phylobetadiversity, is similar to a standard metric of beta diversity, but it takes the additional component of evolutionary relatedness into account (Graham \& Fine 2008). In essence, phylobetadiversity allows us to incorporate a time component into our metric of beta diversity. We use this novel approach to investigate the previously unsampled armored scale insect diversity in a tropical rainforest. 


\section{MATERIALS AND METHODS}

\section{Experimental Design}

Armored scale insects were collected from two sites in the Daintree Rainforest of Australia, including (1) an older, well-established rainforest canopy and (2) and an adjacent, 5year old re-vegetation plot with similar host plant constituency. Due to the fact that female Diaspidids (the most frequently encountered life stage) are sessile, traditional methods for insect collection such as fogging or sweep netting are not efficacious. Therefore, insects must be collected by hand from leaves and brought to the lab for processing. Trees in both sampling sites were mapped and tag with a species-specific identification number, allowing us to trace back insect samples to their host. Individual trees were assigned a random number, sorted by species and within species accordingly. These randomly assigned numbers were used as "rank orders" within each tree species, allowing us to place them into rounds. For example, round 1 included the first-ranked individual tree from each species present in the sampling area, round 2 including the second, etc. Therefore, in round 1, we sampled from one individual of every host species present. In round 2, a second individual was sampled (from species with $>1$ individual), etc. When a given tree was found to be dead, inaccessible, or knocked down, we moved on to the next tree in that round.

At each individual, a ten-minute long search was conducted and leaves or leaf-cut outs containing specimens were collected. The length of the branch that was searched within this 10minute period was recorded to approximate the amount of area covered during sampling. A $20 \mathrm{~cm}$ long twig sample and approximately a $20 \mathrm{~cm}^{2}$ bark sample was also collected. Plant material was assigned a unique lot number and associated with the host species on which it was 
found. The plant material was then processed under a dissecting microscope, where excess foliage was discarded, and armored scale insects were placed into a tube containing $100 \%$ ethanol for preservation. Each individual was provided with a unique lot number that could be used to

\section{Canopy Sampling}

Trees within the rainforest canopy plot were accessed using a canopy crane access system (Figure 1). The crane measures $47 \mathrm{~m}$ high and can rotate a full $360^{\circ}$ and extending to a 55 meter radius from its center. Thus, approximately 1 hectare of forest is accessible using this crane system.

\section{Ground Sampling}

The ground survey conducted in the adjacent 5-year old re-vegetation plot (Figure 1) was necessarily more opportunistic, as collection was conducted on foot. Therefore, the number of trees with accessible foliage for sampling was reduced. Moreover, some of the nascent trees in the plot were not yet identified, as they had not been present during the mapping and tagging process in earlier years. However, for all plants with accessible foliage, the sampling strategy and protocol was identical to that conducted in the canopy. 


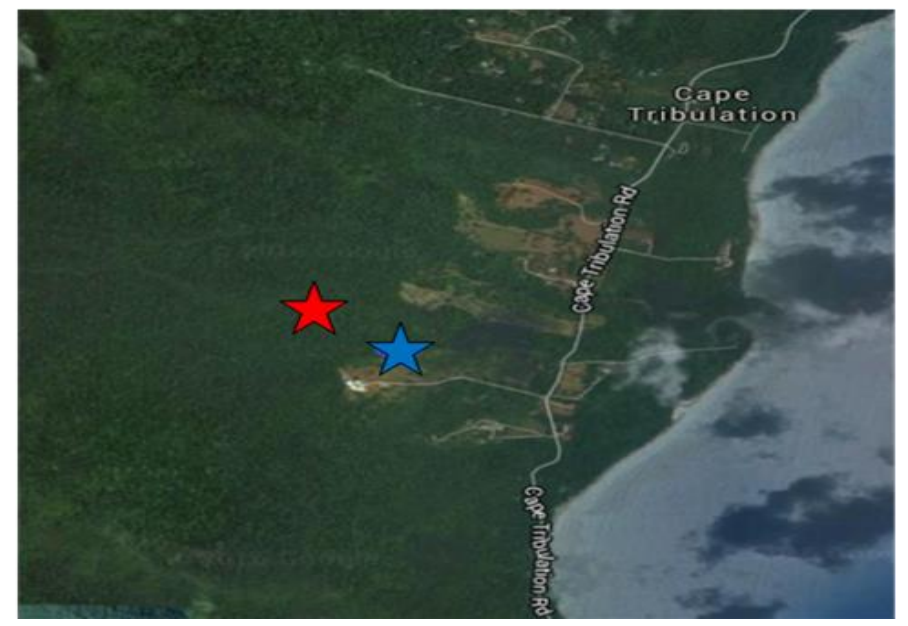

Shapiro 11

Figure 1. Map of sampling sites within the Daintree rainforest of Australia. The red star denotes the location of the canopy sampling using the Daintree Research Observatory canopy crane access system. The blue star denotes the location of ground sampling within the 5-year-old revegetation plot.

\section{Molecular Methods}

Genomic DNA from environmental samples was isolated using a non-destructive preparation technique and utilizing a Qiagen DNeasy extraction kit. PCR was used to amplify three genes: (1) cytochrome oxidase I\&II (COI-II), a mitochondrial protein coding gene, (2) Elongation factor 1- $\alpha(E F 1-\alpha)$, a nuclear protein coding gene, and (3) the nuclear large ribosomal subunit gene (28s). Amplified DNA was sent to Eton Biosciences Inc. for sequencing. Sequences were obtained from 218 environmental taxa and editing was performed using Geneious v7 (Kearse et al. 2012), and environmental sequences were aligned using the algorithm implemented in the program MUSCLE (Edgar, 2004) with a total of 196 known armored scale taxa identified and published (Morse \& Farrell 2006) in GenBank (Benson et. al. 2005). 


\section{Phylogenetic Analysis}

Phylogenetic reconstruction was performed in a Bayesian framework using MrBayes v3.2 (Ronquist et al. 2012). Hypervariable regions of 28S, the intervening tRNA region within COI-II, and the introns within EF1a were excluded from analysis. Protein coding loci (COI-II and EF1- $\alpha$ ) were analyzed using a partitioned framework, with different parameters available for each of the three codon positions. Each partition was analyzed using a general-time-reversible model, chosen a priori. A fraction of these sites were assessed as invariable, and the remaining were allowed to evolve based on a gamma distribution $(\mathrm{GTR}+\mathrm{I}+\mathrm{G})$. This allowed for a more complex Bayesian meta-analysis to be performed using all three loci simultaneously. Markov Chain Monte Carlo methods using 2 chains were allowed to run for 15,000,000 generations, with the first $25 \%$ of tree topologies eliminated as burn-in. Numbers on nodes indicate the Bayseian posteriors (as a fraction out of 1) from the post burn-in samples as a metric of support. Due to the relatively recent evolution of armored scale insects (Gullan and Kosztarab 1997), a standardized branch length of 0.2 was used for species delimitation based on the consensus tree.

\section{Morphological Specimen Preservation}

Following DNA extraction, the cuticles of armored scale insect specimens were preserved in water at $20^{\circ} \mathrm{C}$. The "skins" were mounted onto slides using standard procedures. Slides were incubated for several months.

\section{RESULTS}

Alignment and squaring of the matrices resulted in a final analysis of 2582 base pairs from 219 environmental taxa (134 individuals collected in the canopy and 85 individuals sampled from the re-vegetation plot) and 198 previously identified taxa from GenBank (Benson 
et. al. 2005). The partition of EF1 $\alpha$ included 759 base pairs, 1033 base pairs within 28S, and 790 base pairs within COI-II. The consensus tree obtained after the $25 \%$ post-burn in showing the environmental samples scaffolded with previously identified taxa and the posterior probabilities for nodes represented as fractions of 1 , and tips are labeled according to the unique lot numbers assigned to environmental samples or labeled as previously identified species (Figure 2). Comstockiella sabis was used to root the tree (Morse \& Normark 2006). From this phylogeny, species delimitation was performed. None of the environmental samples could be identified as a previously discovered armored scale species. Only one could be identified to a potential known genus. Using a standard branch length of 0.2 , a total of 38 different species "groups" were identified among the environmental samples; they will herein be referred to as "species", but it should be noted that formal taxonomy has not yet been performed on these specimens.

Frequencies for each of 38 species were plotted for the canopy and re-vegetation plots Each of these unique species were assigned a number (sp1, sp2...sp38), and the number of individuals observed in each group were plotted (Figures $3 \& 4$ ). 

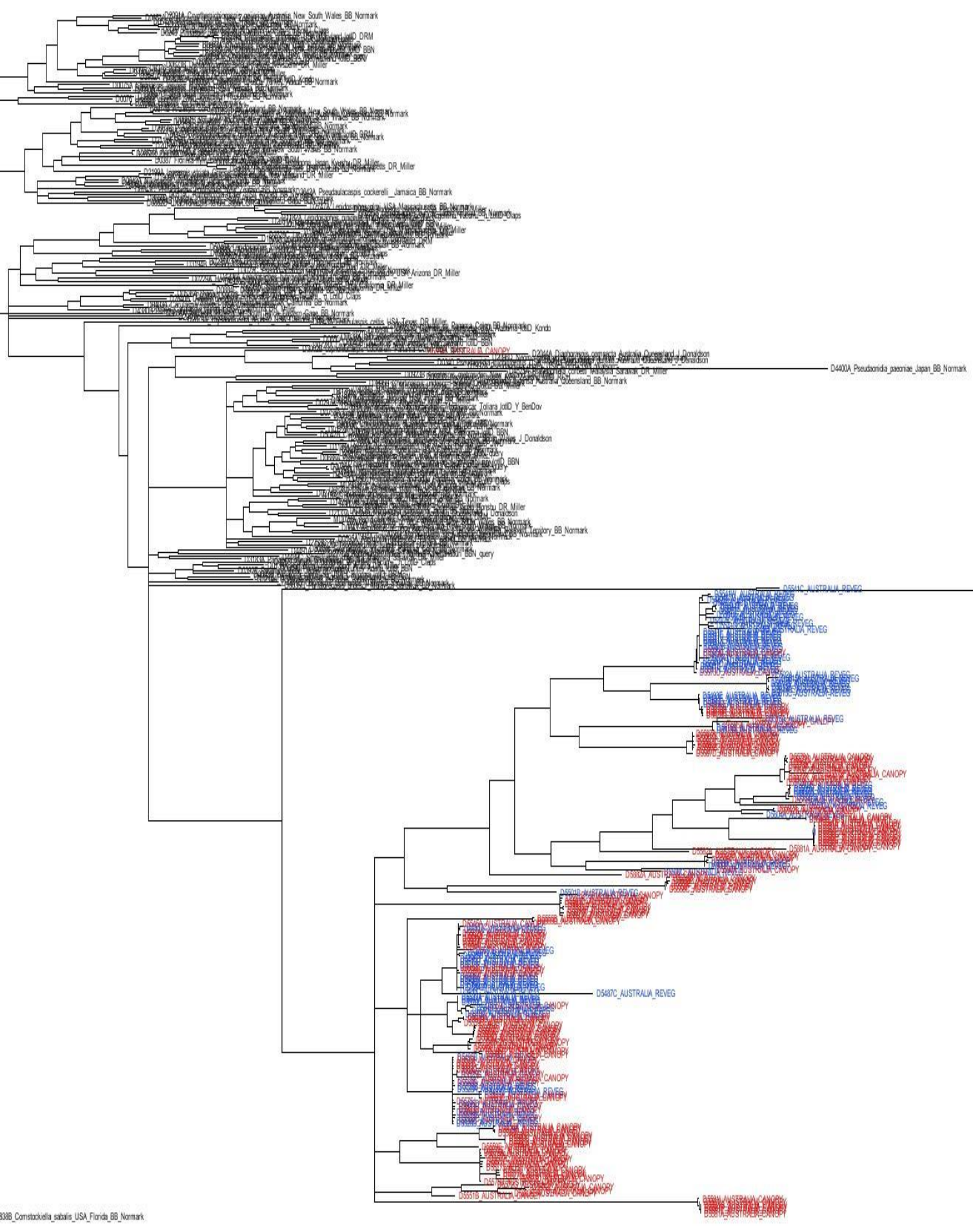

Figure 2. Consensus tree showing previously identified armored scale taxa representing the total known diversity (black), environmental samples collected from the rainforest canopy (red), and environmental samples collected from the ground survey conducted in the re-vegetation plot (blue). Comstockiella sabis was used to root the tree. A branch length of 0.2 was used to differentiate species. 


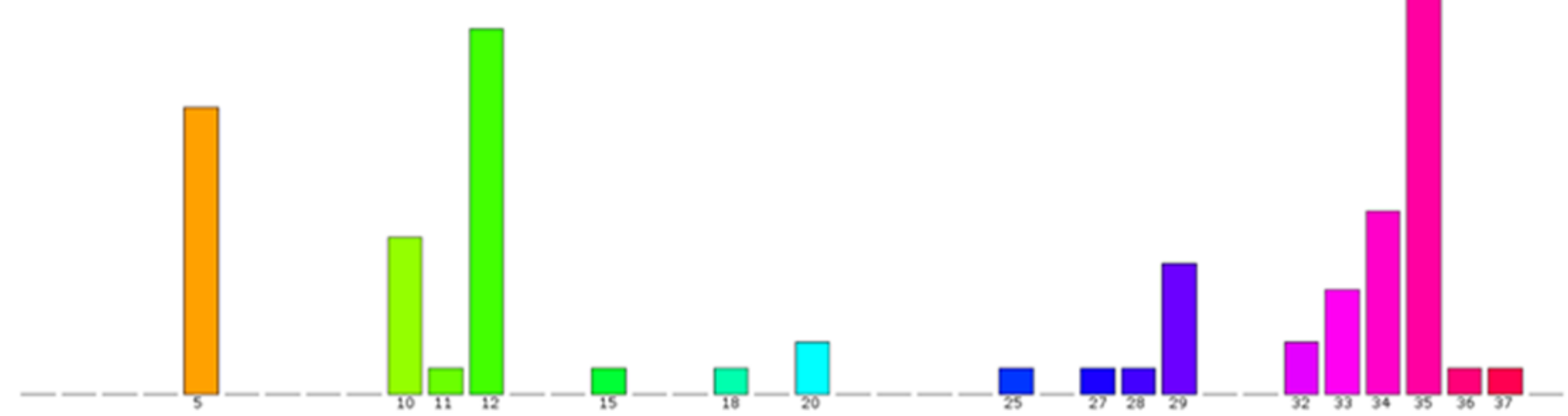

\section{Species Number}

Figure 3. Species frequency (no. of individuals) observed in the re-vegetation plot belonging to each of the 38 species groups observed in the phylogeny.
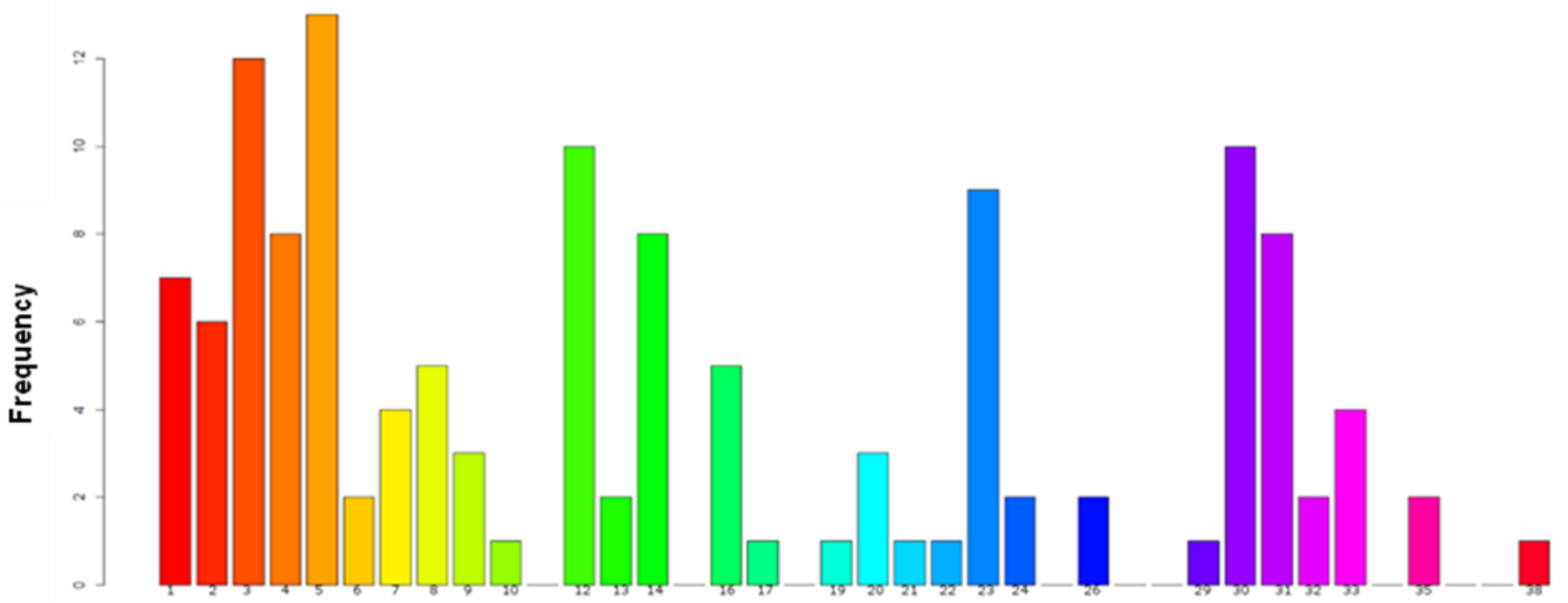

\section{Species Number}

Figure 4. Species frequency (no. of individuals) observed in canopy plot belonging to each of the 38 species groups observed in the phylogeny. 
A collapsed phylogeny was created to perform community data analysis after species delimitation (Figure 5), in which each of the 38 species found is represented by a single tip.

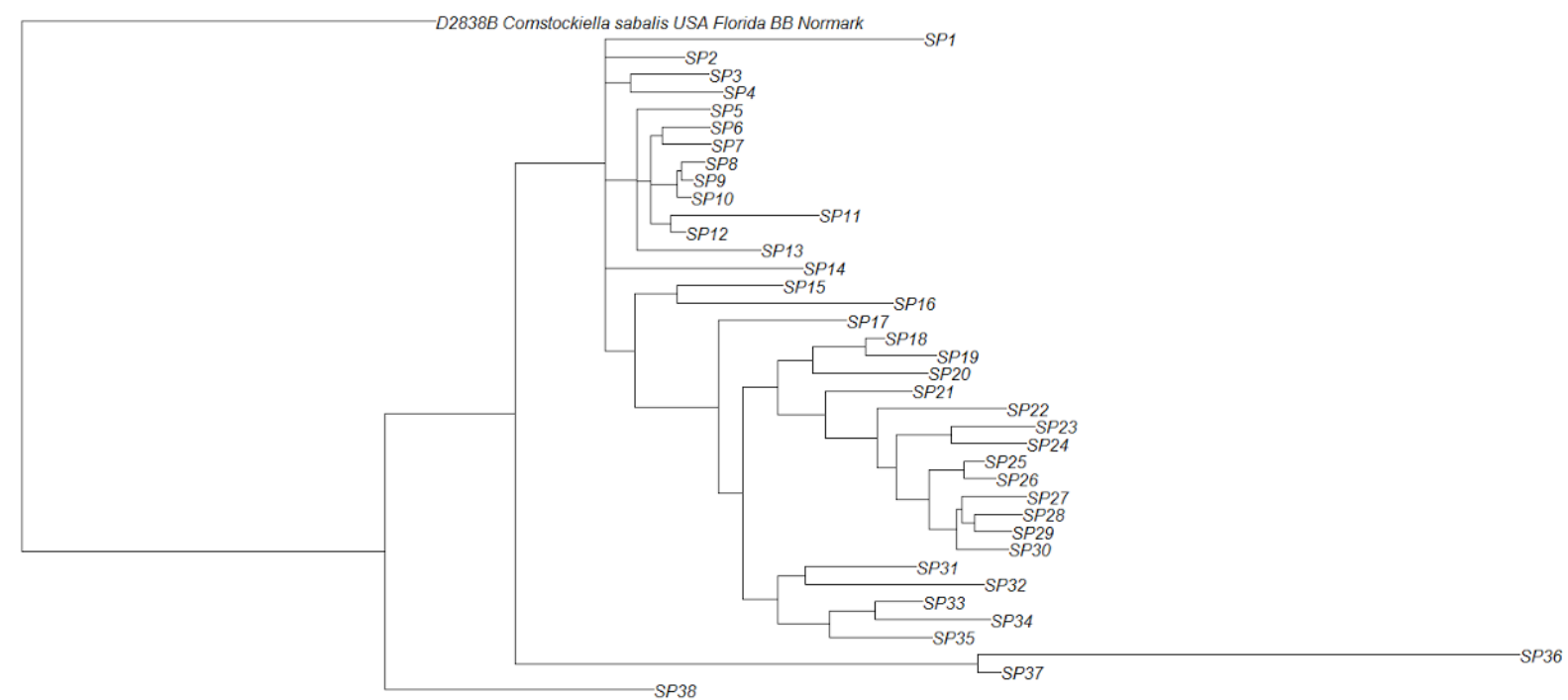

Figure 5. Sub-tree phylogeny of environmental samples, where tips represent a single species (out of with a total of 38 species observed).

Rarefaction curves to analyze the number of species found in each habitat as a function of sample size (Figure 6). This was done to adjust for differences in sample size between the two plots.

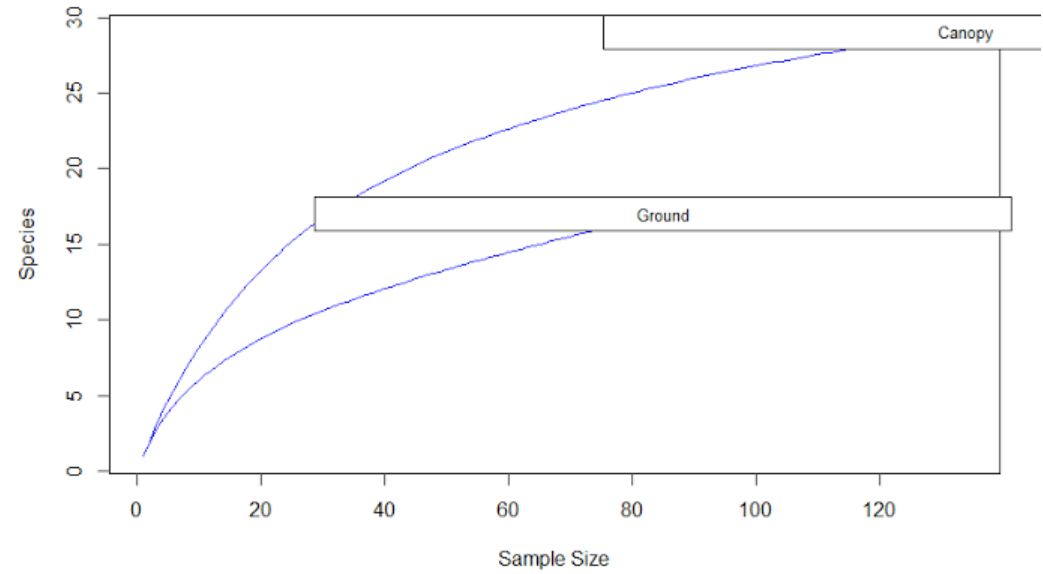

Figure 6. Rarefaction curve to assess species richness as a function of sample size. 
All statistical analyses and figures were performed and created using the 'vegan' and 'picante' packages within Rstudio (Rstudio Team 2015) (Kembel et. al. 2015). Shannon -Weiner diversity indices $\left(\mathrm{H}^{\prime}\right)$ were calculated for the canopy $\left(\mathrm{H}_{\mathrm{c}}{ }^{\prime}=3.07\right)$ and re-vegetation plot $\left(\mathrm{H}_{\mathrm{r}}{ }^{\prime}=2.22\right)$ as a metric of alpha diversity using the following equation (Cadotte et. al. 2010):

1.

$$
\mathrm{H}^{\prime}=\sum_{i=1}^{s} p i \times \ln (p i)
$$

Where $p i$ is the proportion of individuals belonging to the $i t h$ species

Whittaker's index was calculated $\left(\beta_{\mathrm{w}}=0.65\right)$ to estimate general beta diversity (Whittaker 1960) using the following equation in 'picante' (Kembel et. al. 2010):

2 .

$$
\beta_{\mathrm{w}}=\frac{\gamma}{\bar{\alpha}}
$$

Where $\gamma$ is gamma diversity and $\bar{\alpha}$ is mean alpha diversity of individual plots.

Beta diversity is shown visually using a ternary plot (Figure 7), which integrates components a', $b^{\prime}$ and c'.

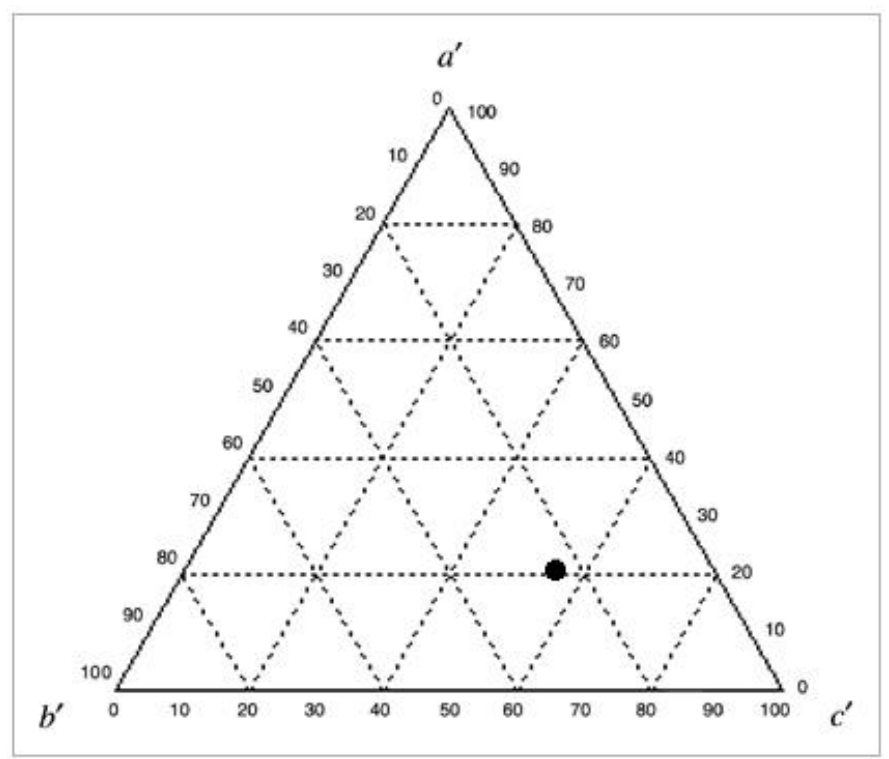

Figure 7: Triangular (ternary) plot displaying beta diversity derived from calculations of Sorenson's dissimilarity index. $\mathrm{A}^{\prime}$ is the richness difference between the sites, $b^{\prime}$ is the percent similarity between the re-vegetation plot and the canopy, and $\mathrm{c}^{\prime}$ is the percent of species replacement between these two habitats (Legendre 2014). 
To address phylobetadiversity, the following phylogenetic species diveristy metrics were calculated using 'picante': Faith's phylogenetic diversity index $\left(\mathrm{PD}_{\text {ground }}=10.65, \mathrm{PD}_{\text {canopy }}=\right.$ 13.15), phylogenegic species richness ( $\mathrm{PSR}_{\text {ground }}=8.22, \mathrm{PSR}_{\text {canopy }}=13.21$ ), phylogenetic species evenness $\left(\mathrm{PSE}_{\text {ground }}=0.37, \mathrm{PSE}_{\mathrm{canopy}}=0.42\right)$, and phylogenetic species clustering $\left(\mathrm{PSC}_{\text {ground }}=0.82, \mathrm{PSC}_{\mathrm{canopy}}=0.77\right)($ Cadotte et. al. 2010).

\section{DISCUSSION}

\section{Characterization of Alpha and Beta Diversity}

It is notable that none of the specimens collected from either sampling plot in the Daintree rainforest could be identified as previously characterized species of armored scale insects. Encounter of new diversity was expected, as armored scale insects have never before been collected in this locality. Through purely qualitative inspection, species frequencies for each of the 38 species found were markedly different between the canopy and the re-vegetation plot. Due to the difference in sample sizes between the two plots (134 individuals collected from the canopy and 85 individuals collected from the ground survey), a rarefaction analysis was used as a visual comparison of species richness, taking into account the unbalanced sampling. After standardization of sample size between the two plots, it was clear that species richness accumulated more rapidly in the canopy than in the re-vegetation plot; this was to be expected under the which was expected under the time-stability hypothesis (Sanders 1969). In further support of this hypothesis, the Shannon-Wiener index, which takes into account both species richness and species evenness, was greater in the canopy $\left(H_{c}^{\prime}=3.07\right)$ than for the re-vegetation 
plot $\left(\mathrm{H}_{\mathrm{r}}{ }^{\prime}=2.22\right)$, showing a greater alpha diversity in the older, established rainforest canopy (Cadotte et. al. 2010).

To address the issue of traditional beta diversity from a pure community-ecology standpoint, Whittaker's index was first calculated, which can take on a value from 0 to 1 (Whittaker 1960), with a value of 0 being indicative of more homogenous habitats. The value obtained $\left(\beta_{\mathrm{w}}=0.65\right)$ is indicative of high dissimilarity in armored scale diversity between the canopy and re-vegetation plots, suggesting a high degree of beta diversity. Beta diversity can be visually represented using a ternary plot (Figure 7), which displays the following components along three axes: a' - species richness difference, $b^{\prime}$ - percent similarity between the two habitats, and $c^{\prime}$ - species replacement, or species turnover between the two plots (Legendre 2014). These components can be used to infer the two major components of beta diversity: species richness/abundance differences, and species turnover (Koleff et. al. 2003). The left side of the ternary plot measures a', or species richness differences as a proportion. In these data, (Figure 7) this value is $20 \%$. Thus, there was a $20 \%$ difference in species richness between the two habitats. The bottom axis of the ternary plot measures b', or the percent similarity between the revegetation plot and the canopy plot. The observed a' value indicates that approximately $20 \%$ of the observed beta diversity is attributable to differences in species richness. The next component, $\mathrm{b}^{\prime}$ indicates nearly $60 \%$ similarity between plots. Although this is a high value of similarity, it is actually remarkably different when considering that the two sampling sites had the constituency of host species. The value of the third component, c', is $20 \%$, representing the percentage of species turnover between the two sites. This third element addresses the component of change involving simultaneous species gains and losses across ecological gradients, leading to habitat filtering (Legendre 2014). 


\section{Addressing Phylobetadiversity}

Though the aforementioned "traditional" metrics of beta diversity are informative on their own, a more in-depth assessment of beta diversity can be obtained after integration of the phylogenetic component. From these metrics, we can observe how biotic factors such as population size interact with largescale evolutionary processes (Graham \& Fine 2008). Faith's phylogenetic diversity index (PD) represents the sum of the branch lengths in a consensus tree, effectively inferring phylogenetic distance. The results indicate that the canopy samples had a longer average phylogenetic difference (branch length) than those samples from the revegetation plot; therefore, canopy samples exhibit greater phylogenetic diversity. Communities exhibiting phylogenetic clustering tend to have lower PD values (Kembel et. al. 2010); thus, we can infer that armored scale insects in the younger re-vegetation plot exhibit more phylogenetic clustering, as the species found in this plot captures only a small part of the total observed diversity in the phylogeny. In contrast, the canopy samples comprise a much larger portion of the total diversity present in the phylogeny. With a higher PD value, there is evidence for greater phylogenetic dispersion in the canopy samples. These results are supported by the time-stability hypothesis (Sanders 1969), as we would expect both greater diversity and greater phylogenetic dispersion in the older, established rainforest canopy as compared to the 5-year-old re-vegetation plot.

Phylogenetic species variability (PSV), which quantifies phylogenetic relatedness among species in a community independent of any trait data (Vamosi et. al. 2009) was nearly the same in both habitats. Phylogenetic species richness (PSR) is the product of PSV and species richness, and this score includes a "penalty" for relatedness of species (Vamosi et. al. 2009).This value was found to be higher in the canopy than in the ground survey. Thus, the canopy exhibited 
greater species richness, even when disregarding the component of homology. Phylogenetic species evenness (PSE) is a metric similar to PSV, but that measures variation among individuals rather than species, utilizing abundance data. This was similar in both habitats, suggesting a rather "even" variance among individuals in both habitats. Finally, phylogenetic species clustering (PSC) allowed us to further measure species relatedness. In concordance with the PD results, the PSC was found to be higher in the re-vegetation plot, providing further evidence for phylogenetic clustering in samples collected from the ground survey and relatively greater phylogenetic dispersion in those samples collected from the canopy (Kembel et. al. 2010).

\section{CONCLUSION}

In conclusion, there is evidence supporting the notion that armored scale insect diversity can, indeed, be modeled according to the tenets derived from island biogeography theory. In particular, the greater observed diversity in the older canopy plot as compared to the revegetation plot was to be expected, in accordance with the time-stability hypothesis (Sanders 1969). This is further supported by the high degree of beta diversity, or community heterogeneity, between the two habitats, despite the fact that they are not geographically distant from one another and that they have the constituency in tree species.

If armored scale insects are truly generalists, we might expect to see a greater amount of habitat homogeneity, or a lower beta diversity, than what was observed. Future studies should complete further analysis utilizing host information. This information would allow us to discern which armored scale taxa can utilize multiple hosts, and also whether or not multiple armored scale taxa were found on a single host in a certain habitat, compared to that same host in a different habitat. Addition of this dimension will allow us to further elucidate the bizarre life 
history of armored scale insects and further our understanding of their diversity in natural habitats.

\section{REFERENCES}

1. Abele, L. G., \& Walters, R. 2016. "The Stability Time Hypothesis: Reevaluation of the Data," no. October 1979. doi:10.1086/283502.

2. Andersen, J.C., Wu, J., Gruwell, M.E., Gwiazdowski, R., Santana S.E., Feliciano, N.M., Morse, G.E., Normark, B., 2010. A phylogentic analysis of armored scale insects (Hemiterpa: Diaspididae), based upon nuclear, mitochondrial, and endosymbiont gene sequences. Molecular Phylogenetics and Evolution 57: 992-1003.

3. Benson, D. A., Karsch-Mizrachi, I., Lipman, D. J., Ostell, J., \& Wheeler, D. L. 2005. GenBank. Nucleic Acids Research 33: 34-38.

4. Bray, J. R. \& Curtis, J. T. 1957. An ordination of upland forest communities of southern Wisconsin. Ecological Monographs 27:325-349.

5. Cadotte, M.W., Davies, T.J., Regetz, J., Kembel, S.W., Cleland, E., \& Oakley, T.H. 2010. "Phylogenetic Diversity Metrics for Ecological Communities: Integrating Species Richness, Abundance and Evolutionary History." Ecology Letters 13 (1): 96-105.

6. Cavender-Bares, J., Kenneth H. K., Fine P.V.A, \& Kembel, S.K. 2009. "The Merging of Community Ecology and Phylogenetic Biology.” Ecology Letters 12 (7): 693-715.

7. Edgar, R.C. 2004. MUSCLE: multiple sequence alignment with high accuracy and high throughput. Nucleic Acids Research 32(5), 1792-1797.

8. Graham, Catherine H., and Paul V. A. Fine. 2008. "Phylogenetic Beta Diversity: Linking Ecological and Evolutionary Processes across Space in Time.” Ecology Letters 11 (12): 1265-77.

9. Gullan, P.J., \& Kosztarab, M. 1997. Adaptations in Armored Scale Insects. Annual Review of Entomology 42: 23-50.

10. Kearse, M., Moir, R., Wilson, A., Stones-Havas, S., Cheung, M., Sturrock, S. , Buxton, S., Cooper, A., Markowitz, S., Duran, C., Thierer, T., Ashton, B., Mentjies, P., \& Drummond, A. 2012. Geneious Basic: an integrated and extendable desktop software platform for the organization and analysis of sequence data. Bioinformatics, 28(12), 1647-1649.

11. Kembel, S. W., P. D. Cowan, M. R. Helmus, W. K. Cornwell, H. Morlon, D. D. Ackerly, S. P. Blomberg, \& Webb, C. O. 2010. "Picante: R Tools for Integrating Phylogenies and Ecology. ” Bioinformatics 26 (11): 1463-64.

12. Koleff, P., Gaston, K. J. \& Lennon, J. J. 2003. Measuring beta diversity for presenceabsence data. Journal of Animal Ecology, 72: 367-382.

13. Legendre, Pierre. 2014. Interpreting the Replacement and Richness Difference Components of Beta Diversity. Global Ecology and Biogeography 23.11: 1324-334.

14. MacArthur, R. H. \& Wilson, E. O. 1967. The theory of island biogeography. Princeton, NJ: Princeton University Press. 203. 
15. Miller, D.R., Miller, G.L, Hodges, G.S., \& Davidson J.A. 2005. Introduced scale insects (Hemiptera: Coccoidea) of the United States and their impact on US agriculture. Proc. Entomol. Soc. Wash 107: 123-158.

16. Morales, M.G., Denno, B.D., Miller, D.R., Miller, G.L., Ben-dov, Y., \& Hardy, N.B. 2016. Database Update ScaleNet : A Literature-Based Model of Scale Insect Biology and Systematics 5 (1): 1-5.

17. Morse GE, Normark B, 2006. A molecular phylogenetic study of armoured scale insects (Hemiptera: Diaspididae). Systematic Entomology 31: 338-349.

18. Morse, G.E. \& Normark, B.B. 2006. A phylogenetics analysis of armored scale insects (Hemiptera: Diaspididae), based upon nuclear, mitochondrial, and endosymbiont gene sequences. Molecular Phylogenetics and Evolution 57(3): 992-1003.

19. Normark, Benjamin B. 2004. "The Sex Lives of Scales."

20. Normark, B.B., \& Johnson, N.A.. 2011. Niche Explosion. Genetica 139 (5): 551-64.

21. Novotny, V., Basset, Y., Miller, S.E., Weiblen, G.D., Bremer, B., Cizek, L., \& Drozd, P. 2002. Low host specificity of herbivorous insects in a tropical forest. Nature 416: 841844.

22. Ronquist, F., Teslenko, M., Van der Mark, P., Ayres, D.L., Darling, A., Hohna, S., Larget, B., Liu, L., Suchard, M.A., \& Huelsenbeck, J.P. 2012. MrBayes 3.2: Efficient Bayesian phylogenetic inference and model choice across a large model space. Systematic Biology 61: 1-4.

23. RStudio Team (2015). RStudio: Integrated Development for R. RStudio, Inc., Boston, MA URL http://www.rstudio.com/.

24. Sanders, H.L. 1969. Benthic marine diversity and the stability-time hypothesis. Brookhaven Symp. Biol 22: 71-81.

25. Vamosi, S.M., Heard, S.B., Vamosi,J.C. \& Webb, C.O. 2009. Emerging Patterns in the Comparative Analysis of Phylogenetic Community Structure. Molecular Ecology 18 (4): 572-92.

26. Whittaker, R. H. 1960. Vegetation of the Siskiyou Mountains, Oregon and California. Ecological Monographs, 30(3), 279-338. 